\title{
Spectral signature of uncombed penumbral magnetic fields
}

\section{Reply}

\author{
V. Martínez Pillet* \\ Instituto de Astrofísica de Canarias, 38200, La Laguna, Tenerife, Spain
}

Received 11 December 2000 / Accepted 13 December 2000

\begin{abstract}
A combination of two penumbral models similar to those used by Martínez Pillet (2000) is presented. One matches the observed rms fluctuations perfectly while the other reproduces the observed Net Circular Polarization. No factor three mismatch exists in this case. The rationale of using two different, but strongly coupled, models is explained.
\end{abstract}

Key words. Sun: sunspots - Sun: magnetic fields

This paper discusses the criticism by Sánchez Almeida (2001, this issue; SA) to the paper on penumbral magnetic fields published by Martínez Pillet (2000; MP). The two observed magnitudes that are of importance are the Net Circular Polarization $\left(\mathrm{NCP}^{1}\right)$ and the pixel-to-pixel fluctuations of the gradient with height of the (cosine) magnetic field inclination. This last quantity will be referred as the rms fluctuations. The "uncombed" penumbral model built by MP consist of a background magnetic field in which more horizontal penumbral flux tubes are embedded. The background and the tubes have a field-aligned flow with different velocities. The inclination and velocity jumps at the tube boundaries were found by MP to reproduce the observed NCP of the Fe I 6301 and $6302 \AA$ lines.

This penumbral model does not provide information on the rms fluctuations by itself; that would require a more complex model including other tubes in nearby pixels. To estimate the rms fluctuations, MP considered how different realizations, of a more schematic model that preserves key ingredients (see below), could account for the observed magnitude of the fluctuations and chose to use a "random $z_{\mathrm{t}}$ model" in which the height of penumbral tubes was randomly varied to estimate the rms fluctuations (see MP). Key parameters that were included are the spatial resolution of the observations, the size of the jump in magnetic field inclination at the tube boundaries, the tube diameter and a weighting function, $g(z)$. With the parameters used by MP, the value of the rms fluctuations was found to be a factor three larger than the observed one.

\footnotetext{
* e-mail: vmp@ll.iac.es

1 The wavelength integral of the Stokes V profile.
}

The mismatch of a factor three was considered as quite reasonable by MP but is now criticized by SA. We have therefore generated a new combination of two models that together reproduce the NCP at disk center and match the rms fluctuations $\left(1.410^{-4} \mathrm{~km}^{-1}\right)$. This model has filling factor unity, a jump in inclination of $15^{\circ}$ (MP used $30^{\circ}$ ), background flow velocity of $-3 \mathrm{~km} \mathrm{~s}^{-1}$ (MP used $-1 \mathrm{~km} \mathrm{~s}^{-1}$ ), and tube flow velocity of $-5 \mathrm{~km} \mathrm{~s}^{-1}$ (MP used $-2 \mathrm{~km} \mathrm{~s}^{-1}$ ). The smaller jump in inclination is fully compensated by larger flow speeds. Contrary to what is stated in SA, almost any NCP value can be reproduced after matching (in the random $z_{\mathrm{t}}$ model) the rms fluctuations by simply changing the flow speeds (which do not enter in the rms estimate).

The use of two models is criticized by SA. We note that the key ingredients from the models that use radiative transfer to compute the NCP are all passed consistently to the random $z_{\mathrm{t}}$ model, except for the half width of the weighting function $g(z)$ which is not self-consistently computed from the radiative transfer calculations (but note that the same applies to the $L_{z}$ parameter used in the weighting function, $g(z)$, of Sánchez Almeida 1998). However, the $200 \mathrm{~km}$ width used by MP is a perfectly reasonable value that corresponds to the size of the region where penumbral tubes are very efficient in generating NCP (in the range of $1.2-1.6 \mathrm{~m} \AA$ for Fe I $6302.5 \AA$ ).

The rms fluctuations are not directly accessible to observations. They are derived from the null divergence condition applied to vector magnetograms. Comparison with Sánchez Almeida (1998) therefore leads to the question 
whether the $\nabla \cdot \boldsymbol{B}=0$ condition can be satisfied in the radiative transfer model that reproduces the NCP. As shown already by MP (his Eqs. (3) and (4)), this condition only demands having a purely azimuthal field vector $B \approx B_{\phi}$ when the background field lines wind around the penumbral flux tubes. This is a perfectly reasonable scenario.

Our schematic uncombed model points to the fact that a penumbra consisting of $100 \mathrm{~km}$ size flux tubes cannot be ruled out. Note that none of the two penumbral scenarios studied by Sánchez Almeida (1998) represents this case.

\section{References}

Martínez Pillet, V. 2000, A\&A, 361, 734 (MP)

Sánchez Almeida, J. 1998, ApJ, 497, 967

Sánchez Almeida, J. 2001, A\&A, 369, 643 (SA) 